Individual differences in the $P_{D}$ component support object-file updating as the source of the samelocation cost in attentional orienting

\author{
Claire Bradley ${ }^{1+*}$, Anthony M. Harris ${ }^{1+*}$, Sera Yijing Yoo ${ }^{2}$, Jason B. Mattingley ${ }^{1,2,3}$ \\ ${ }^{1}$ Queensland Brain Institute, The University of Queensland, Brisbane, Queensland, Australia \\ ${ }^{2}$ School of Psychology, The University of Queensland, Brisbane, Queensland, Australia \\ ${ }^{3}$ Canadian Institute for Advanced Research (CIFAR), Toronto, Ontario, Canada
}

+ These authors contributed equally to this work.

* Corresponding authors. Correspondence should be addressed to: claire.bradley@uq.edu.au or anthmharris@gmail.com

Acknowledgements: This work was supported by the Australian Research Council Centre of Excellence for Integrative Brain Function (ARC Centre Grant CE140100007). CB was supported by a University of Queensland post-doctoral researcher fellowship (UQ Research Stimulus Allocation Two (Fellowships)). AMH was supported by an ARC Discovery Early Career Researcher Award (DE220101019). JBM was supported by an NHMRC Leadership Award (GNT2010141). 


\begin{abstract}
Spatial cues that mismatch the colour of a subsequent target have recently been shown to slow responses to that target. The source of this 'same location cost' (SLC) is currently unknown. Two potential sources are attentional signal suppression and object-file updating. Here, we tested these accounts by reanalysing data from a previously published spatial-cueing study in which we recorded brain activity using electroencephalography (EEG), and focusing on the event-related $P_{D}$ component, which is thought to index attentional signal suppression. Correlating $P_{D}$ component amplitude with SLC magnitude gives rise to two opposing predictions. If attentional signal suppression is the source of the SLC, then the SLC should be positively correlated with $P_{D}$ amplitude. Alternatively, if object-file updating is the source of the SLC, the SLC should be negatively correlated with $P_{D}$ magnitude, as a more suppressed signal should be easier to update with subsequent target features. Forty-eight participants performed a colour-based spatial-cueing task, and showed a pattern of reaction times consistent with an SLC. Across participants, SLC and PD magnitudes were negatively correlated $(r=-.41$, $p=.004)$. This finding is not compatible with an attentional suppression account of the SLC, and instead supports object-file updating as the source of the SLC.
\end{abstract}

Keywords: Same location cost, attentional capture, spatial cueing, attention, object-file updating 


\section{Individual differences in the $P_{D}$ component support object-file updating as the source of the same- location cost}

Human visual attention is remarkably flexible in the ways it can prioritise particular stimulus properties during task performance. Attention can be tuned to respond to particular target features (e.g., red or green; Folk et al., 1992; Folk \& Remington, 1998; Harris et al., 2013), feature relations (e.g., redder, smaller, etc., Becker, 2010; Becker et al., 2010, 2013, 2014), feature singletons (e.g., Bacon \& Egeth, 1993; Harris et al., 2015, 2019), or conjunctions of relations and/or features (e.g., Becker et al., 2017; 2020). Numerous studies have shown that when participants search for a target with a particular stimulus property, cues presented prior to the search array capture attention to their location if they possess the target property. Such attentional capture manifests as a 'same location benefit' (Carmel \& Lamy, 2014), such that responses to a subsequent target are speeded if the target shares the location of the cue (i.e., attentional capture). However, many studies have also demonstrated a 'same location cost' (SLC) produced by cues that do not match the target properties (e.g., Carmel \& Lamy, 2014, 2015; Harris et al., 2013, 2017; Lamy et al., 2015; Schoeberl et al., 2018; Travis et al., 2019). The mechanisms underlying the SLC are currently unclear, but two potential sources are object-file updating (Carmel \& Lamy, 2014) and attentional suppression of nontarget cues (Gaspelin \& Luck, 2018b).

Object file theory proposes that when a stimulus is presented, a temporary episodic represention of this stimulus is formed as an 'object file' (Kahneman et al., 1992), in which the properties of that object (size, colour, shape, position, etc.) and their successive states are stored. The object-file updating account of the SLC (Carmel \& Lamy, 2014) proposes that response delays for targets cued by nontarget features are due to the time costs involved in overwriting cue-related feature information at the target location with the features of the target. This initial proposal was supported by an experiment in which participants performed a spatial cueing task, either with or without placeholders highlighting possible stimulus locations. With placeholders creating continuity between the cues and targets, and thus theoretically favoring the creation of an object file, a robust SLC was observed at all cue-target intervals tested. Without placeholders, the SLC was smaller and less consistently different from zero across cue-target intervals. Carmel and Lamy (2014) concluded that, without the placeholders, there was no consistent object to attach features to, and so nothing to be updated upon appearance of the target.

An alternative account of the SLC is that attention actively suppresses nontarget features (Lamy et al., 2004; Eimer et al., 2009). Recently, studies have shown that features that regularly appear as search distractors can be suppressed (e.g., Chang \& Egeth, 2019; Vatterott \& Vecera, 2012), receiving less attention than irrelevant background items (Gaspelin \& Luck, 2018b). For example, Gaspelin and colleagues have shown that salient distractors presented during visual search receive fewer fixations than irrelevant background items (Gaspelin et al., 2017). Using a secondary memory task, Gaspelin and colleagues also showed that when memory probes are presented at the search locations, recall is worse for items presented at the distractor location than at other locations (Gaspelin et al., 2015). Together, these results suggest that attention is suppressed at the location of known distractors. On this account, presenting cues in a colour regularly associated with nontarget items produces attentional suppression at the location of the cue, thus giving rise to the SLC.

One way of assessing attentional suppression directly in cueing tasks, without relying on memory probes, which could exert unknown influences on attentional control, is by recording brain activity during task performance using electroencephalography (EEG). The $P_{D}$ component of the EEG signal is thought to provide an index of attentional suppression (Hickey et al., 2009; Sawaki \& Luck, 2010). The $P_{D}$ component is a positivity in posterior electrodes contralateral versus ipsilateral to the location of a 
suppressed stimulus (e.g., a distractor). $P_{D}$ components, for example, are produced contralateral to the location of a singleton distractor in visual search, and their amplitude is correlated with the extent of memory probe suppression (Gaspelin \& Luck, 2018a). Thus, the $P_{D}$ component provides an index of attentional inhibition that can be related to behaviour.

Here we aimed to determine the source of the SLC by testing competing predictions arising from the object-file updating and attentional suppression accounts. We used data from one of our previous spatial cueing studies (Harris et al., 2017), in which participants ( $N=48)$ reported the orientation of a target of a particular colour that was presented among coloured distractors. Nonpredictive cues were presented in target and non-target colours. We correlated each individual's SLC with the magnitude of their $\mathrm{P}_{\mathrm{D}}$ component evoked by nontarget-coloured cues. If the SLC is produced by attentional suppression, then stronger suppression (indexed by a larger $\mathrm{P}_{\mathrm{D}}$ component) should be positively correlated with the magnitude of the SLC. If the SLC is produced by object-file updating, however, there should instead be a significant negative correlation between the $P_{D}$ component and the SLC, as stronger attentional suppression of the cue should produce a weaker object-file representation which consequently should be more readily updated.

\section{Methods}

The current work reports a reanalysis of data previously published by our group. The full details of that study, which focussed on the roles of EEG oscillations in attentional capture, can be found in Harris et al. (2017). Data were combined from two almost identical protocols, as the Bayes Factor for a between-subjects comparison across experiments revealed strong evidence for no behavioural difference between them $\left(\mathrm{BF}_{01}=14.7\right)$.

\section{Participants}

48 participants (aged 18-31 years, mean $=22.22$ years, 26 females) completed the study and provided analysable data. All participants were right-handed, had normal or corrected-to-normal vision, provided written informed consent and were monetarily compensated for their time. The study was approved by The University of Queensland Human Research Ethics Committee.

\section{Behavioural Task}

Participants completed a spatial cueing task (Folk et al., 1992) (Figure 1). On each trial, participants fixated a central grey cross surrounded by four grey placeholder circles (500-900ms), followed by a cue display $(70 \mathrm{~ms})$, in which all four placeholder circles doubled in thickness, and one changed colour (the cue). The cue was equally likely at each location, and could be either red, green, blue, yellow, or grey (no cue), with equal likelihood. The fixation display was then presented again (130ms), followed by the target display $(100 \mathrm{~ms})$, in which four ' $T$ ' stimuli appeared, one within each of the placeholder circles. Each ' $T$ ' stimulus was a different colour (red, blue, green, or yellow). Two of the 'Ts' were always rotated $90^{\circ}$ to the right, two always rotated $90^{\circ}$ to the left. Participants were required to make a speeded response to the orientation of the target-coloured ' $T$ '. In Experiment $1(n=24)$ the target was always red, whereas in Experiment 2, the target colour was counterbalanced between all four colours across participants $(n=24)$. Participants were then presented with the fixation display for $1500 \mathrm{~ms}$, during which they could make a response. Cue and target locations were uncorrelated, each being equally likely at all locations, such that the location of the cue provided no information about the location of the subsequent target. Participants completed 1040 experimental trials, plus 80 practice trials. 
Figure 1.

Example Stimulus Sequence

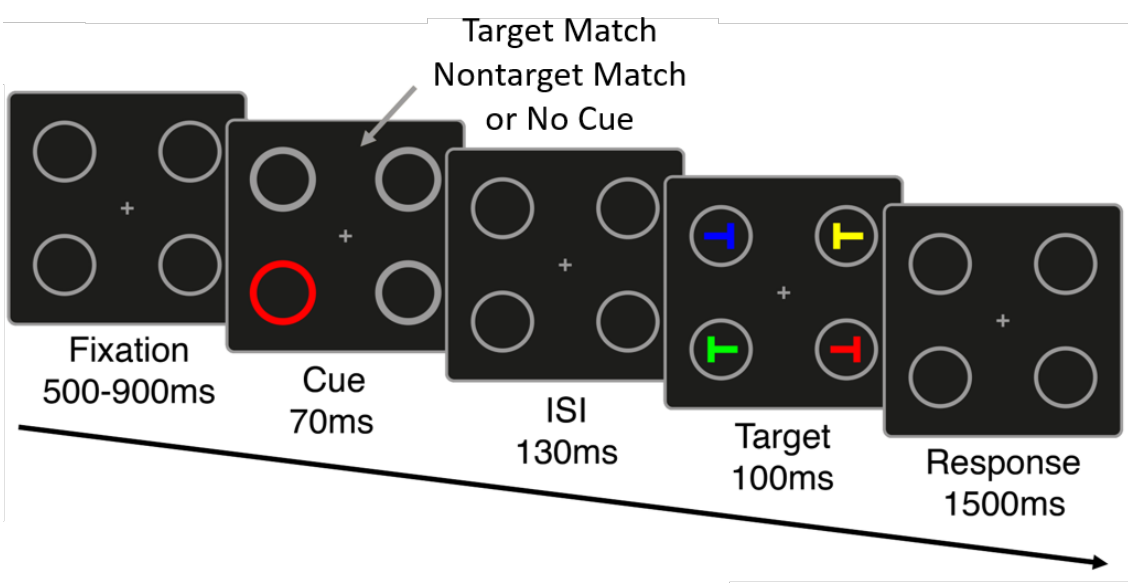

Note. Participants fixated centrally and reported whether the target-coloured " $T$ " was rotated to the right or left. Prior to target onset, a cue was presented that matched either the target colour or one of the non-target colours (or no cue was presented). The cue was equally likely at all locations, and was therefore not predictive of the subsequent target location.

\section{EEG recording and analysis}

EEG data were recorded using a BioSemi Active Two system, digitized at a rate of $1024 \mathrm{~Hz}$. We recorded from 64 active $\mathrm{Ag} / \mathrm{AgCl}$ scalp electrodes arranged according to the 10-10 system (Chatrian et al., 1985), with Common Mode Sense and Driven Right Leg electrodes as ground, and Common Mode Sense as reference.

Offline EEG pre-processing was performed with the EEGLAB Toolbox (Delorme \& Makeig, 2004) using custom-written MATLAB scripts. Data were high-pass filtered at $0.3 \mathrm{~Hz}$ and re-referenced to the average of all scalp electrodes. Trial epochs were extracted between $-800 \mathrm{~ms}$ and $2000 \mathrm{~ms}$ relative to cue onset. Trials containing muscle artifacts or eye movements were rejected by visual inspection, resulting in an average loss of $<1 \%$ of trials per participant. The data were then further cleaned by means of Independent Components Analysis (ICA), using the SASICA plugin for EEGLAB (Chaumon et al., 2015).

For event related potential (ERP) analysis, the pre-processed EEG data were contralateralised relative to the location of the cue, further epoched between $-100 \mathrm{~ms}$ and $600 \mathrm{~ms}$ relative to the onset of the cue, and baseline corrected by subtracting the average voltage between $-100 \mathrm{~ms}$ and $0 \mathrm{~ms}$. ERPs were calculated from symmetrical regions of interest, averaging data from electrodes $\mathrm{P} 7, \mathrm{P} 9$, and PO7, and electrodes P8, P10, and PO8 as described below. The two ERP components of interest were the N2pc and the $P_{D}$ component, with respective time windows of $160-260 \mathrm{~ms}$ and $260-460 \mathrm{~ms}$, consistent with past studies (Harris et al., 2019; Mertes et al., 2016; Sawaki \& Luck, 2013; Schönhammer et al., 2020; Travis et al., 2019). The N2pc (Luck and Hillyard 1997) is an ERP component measured over posterior electrodes, and is charachterised by a more negative deflection of the ERP in contralateral electrodes, than ipsilateral electrodes, relative to the location of a visual stimulus. It is thought to index the allocation of attention. The presence of this component provides confirmation of attentional capture by target-coloured cues. The N2pc component was previously shown in this data in Harris et al. (2017). It is included here for the purpose of individual differences analysis. Both components were calculated 
as the voltage difference between contralateral and ipsilateral electrodes, averaged over their respective time windows.

\section{Statistical analyses}

All statistical analyses were performed in JASP (JASP Team, 2020). Significance level was set a priori to $\alpha=0.05$. Specific tests are presented in the Results for brevity.

\section{Results}

Full behavioural and N2pc analyses can be found in our previous paper (Harris et al., 2017), separately for the two experiments. Here we combined the data from the two experiments to provide adequate statistical power for the individual differences analyses.

\section{Reaction times Analyses}

Reaction times (RTs) were analysed only for trials in which participants responded to the target correctly ( $M=97 \%$ of trials), excluding RTs faster than $250 \mathrm{~ms}$ and slower than $1500 \mathrm{~ms}$ ( $<1 \%$ of data). Errors occurred on $<5 \%$ of trials on average.

A three-way mixed ANOVA was conducted on participants' RTs, with the within-subjects factors Cue Colour (Target Matching, Nontarget Matching) and Validity (Valid, Invalid), and the between-subjects factor of Experiment (Experiment 1, Experiment 2). As expected, there were no significant effects involving the factor of Experiment (all ps $>.11$ ). There was, however, a significant interaction between Validity and Cue Colour, $F(1,46)=283.82, p<.001, \eta^{2}=.09$ (Figure 2). Follow-up tests comparing the effect of Validity for each of the Cue types showed that, as expected, target-coloured cues produced significant attentional capture, $M=50 \mathrm{~ms}, t(47)=15.08, p<.001$, Cohen's $d=2.18$, whereas nontargetcoloured cues produced a significant SLC, $M=-13 \mathrm{~ms}, t(47)=7.03, p<.001$, Cohen's $d=1.01$. Additional analyses confirmed there was no speed-accuracy trade-off (see Harris et al., 2017).

\section{Figure 2}

Reaction times
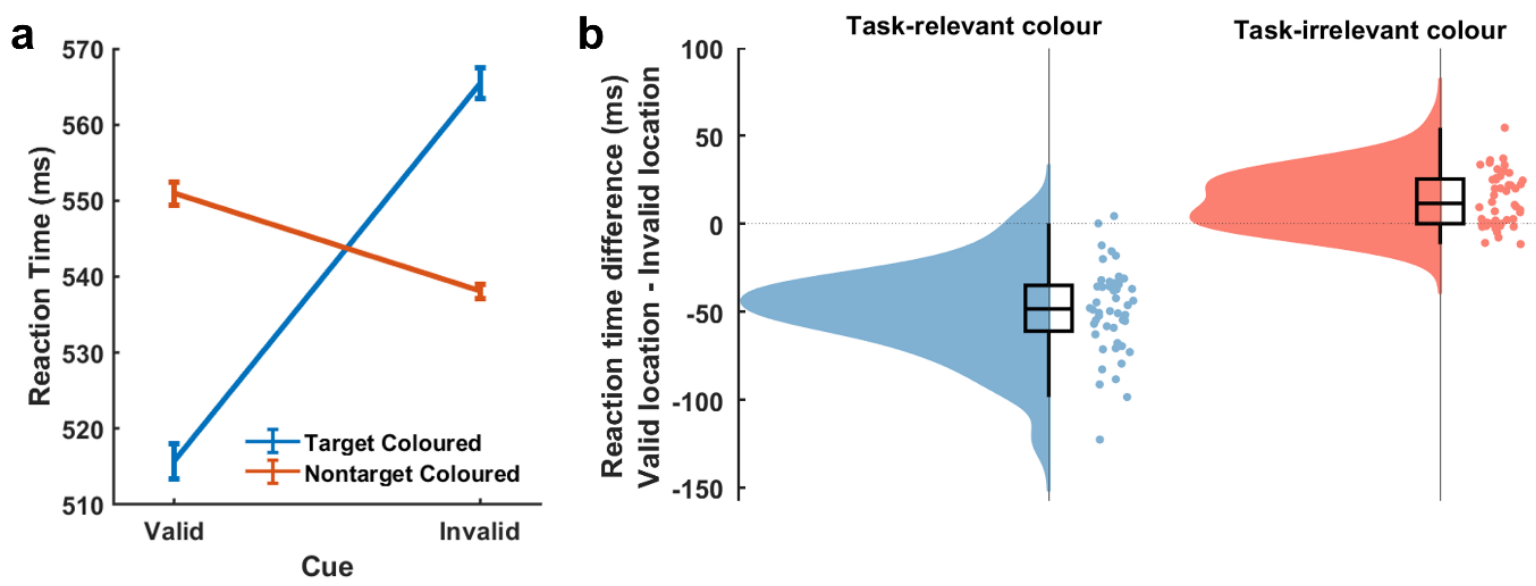

Note. Group level reaction times and individual data for the spatial cueing task. In (a), error bars represent within-participants SEM (Cousineau, 2005; Morey, 2008). In (b), density plots show the distribution of differences between valid and invalid trials across participants. Box plots show median and inter-quartile range. Small dots represent individual participant means. Blue data show the effect 
of target-coloured cues (i.e., attentional capture) and red data show the effect of nontarget-coloured cues (i.e., the SLC).

\section{N2pc Analyses}

A three-way mixed ANOVA was applied to EEG activity averaged within the N2pc timeframe, with within-subjects factors of Cue Colour (Target Matching, Nontarget Matching) and Electrodes (Contralateral, Ipsilateral), and a between-subjects factor of Experiment (Experiment 1, Experiment 2). This revealed a significant interaction between Cue Colour and Electrodes, $F(1,46)=36.70, p<.001$, $\eta^{2}<.01$ (Figure 3). Follow-up paired t-tests revealed a significant N2pc produced by target-coloured cues, $t(47)=5.86, p<.001$, Cohen's $d=0.85$, but no significant N2pc was produced by nontargetcoloured cues, $t(47)=1.94, p=.058$, Cohen's $d=0.28$. Thus, the N2pc results confirm attentional capture by target-coloured cues but not by nontarget-coloured cues.

\section{Figure 3}

\section{Event Related Potentials}

A

A Target Coloured Cues

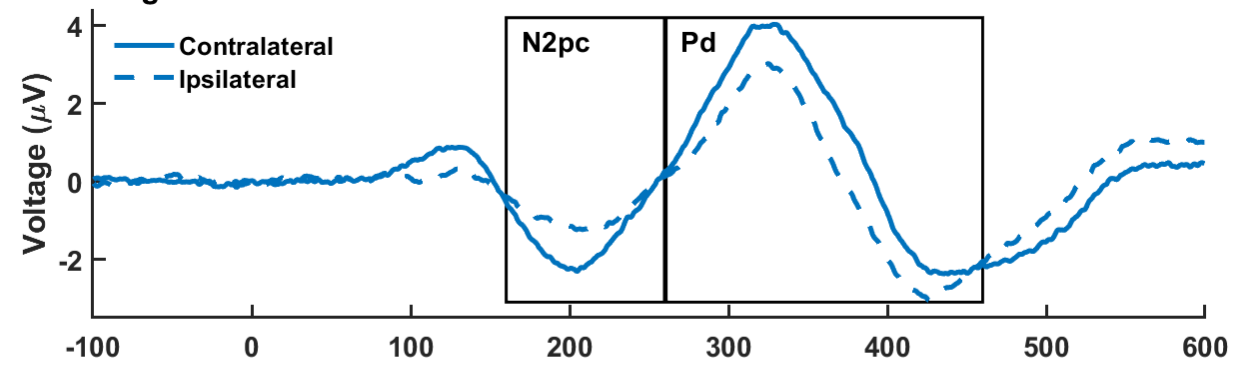

B Nontarget Coloured Cues

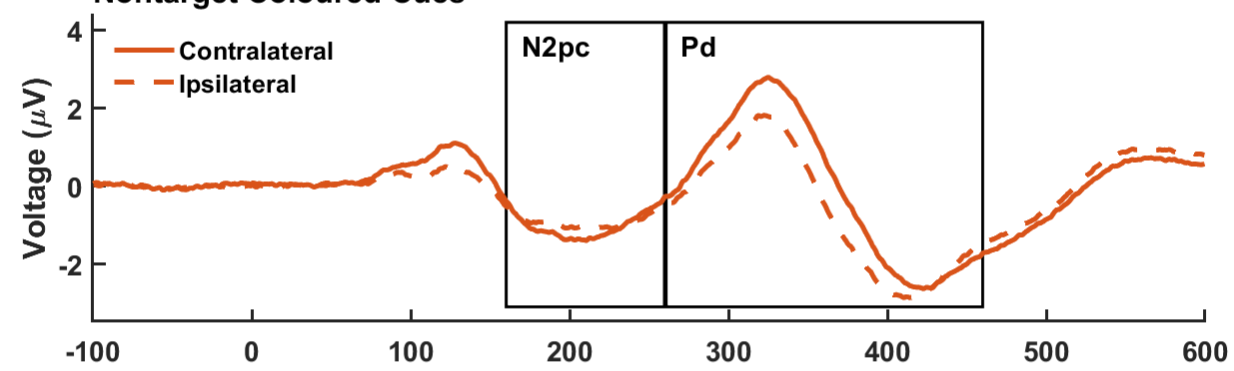

C

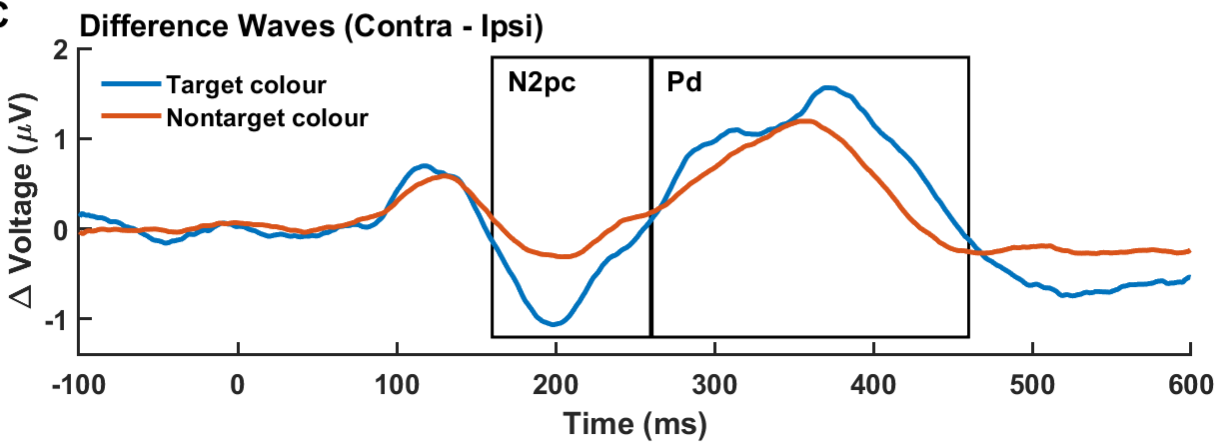

Note. Cue-locked ERPs from focal electrodes contralateral and ipsilateral to the target-coloured (A) and nontarget-coloured (B) cues, as well as their difference waves (C). Cue onset occurred at Oms. Boxes indicate windows for analysis of the $\mathrm{N} 2 p c$ and $P_{D}$ components. 


\section{$P_{D}$ Analyses}

A comparable ANOVA conducted on EEG activity averaged within the $P_{D}$ timeframe revealed a significant interaction between Cue Colour and Electrodes, $F(1,46)=11.70, p=.001, \eta^{2}<.01$ (Figure 3). Follow-up analyses revealed significant $P_{D}$ components produced by both target-coloured cues, $t(47)=8.79, p<.001$, Cohen's $d=1.27$, and nontarget-coloured cues, $t(47)=10.41, p<.001$, Cohen's $d=1.50$. The interaction was driven by a larger $P_{D}$ component following target-coloured cues $(M=$ $0.92 \mu \mathrm{V}, S D=0.73 \mu \mathrm{V})$ than nontarget-coloured cues $(M=0.59 \mu \mathrm{V}, S D=0.39 \mu \mathrm{V}), t(47)=3.45, p=.001$, Cohen's $d=.50$.

\section{Individual Differences}

To test between the two hypotheses about the source of the SLC, we correlated the magnitude of the $P_{D}$ component evoked by nontarget-coloured cues with the magnitude of the SLC in behavioural responses (Figure 4). There was a significantly negative correlation, $r=-.41, p=.004$, indicating that larger amplitude $\mathrm{P}_{\mathrm{D}}$ components were associated with smaller SLC effects in RTs. For the sake of completeness, we also calculated the correlation between the SLC and the magnitude of the N2pc, $r$ $=-.08, p=.573$, and the magnitude of the target-elicited attentional capture effect and both the N2pc, $r=.01, p=.934$, and the $\mathrm{P}_{\mathrm{D}}, r=.08, p=.585$. None of these tests approached statistical significance.

\section{Figure 4}

Correlations Between ERPs and RT cueing effects
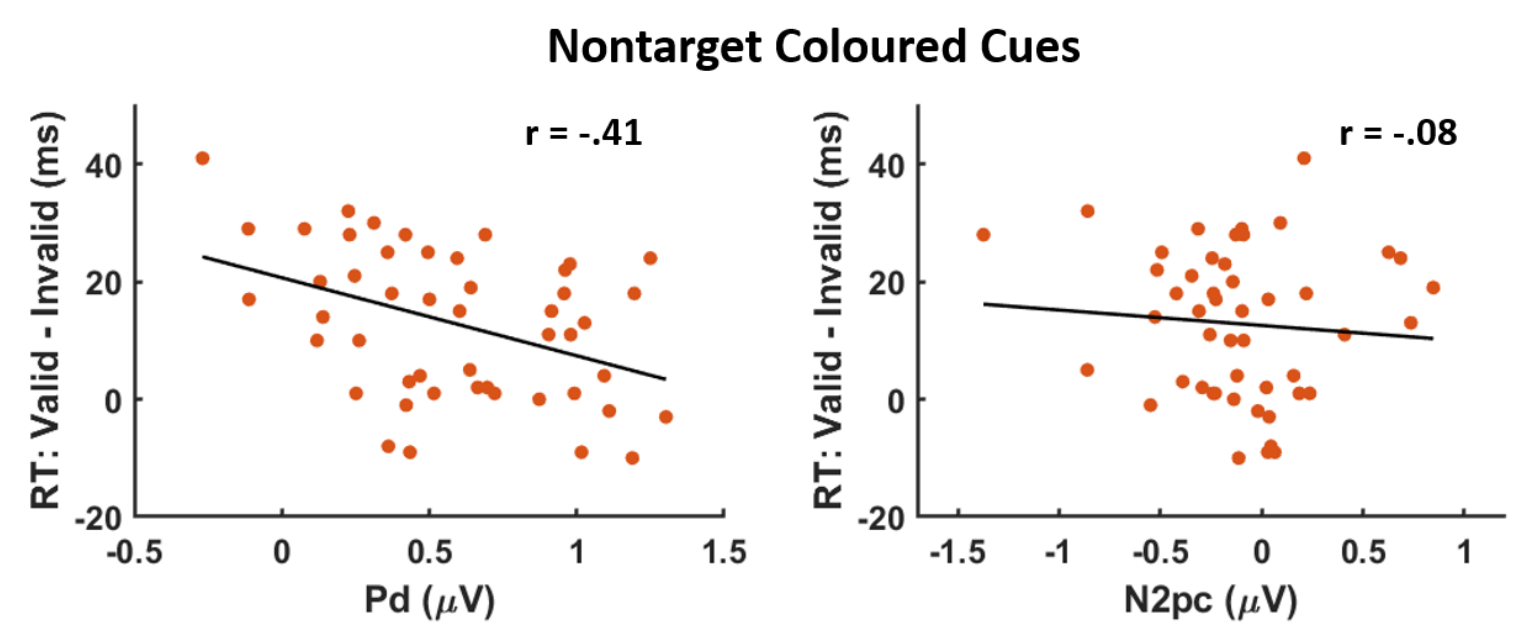

Target Coloured Cues
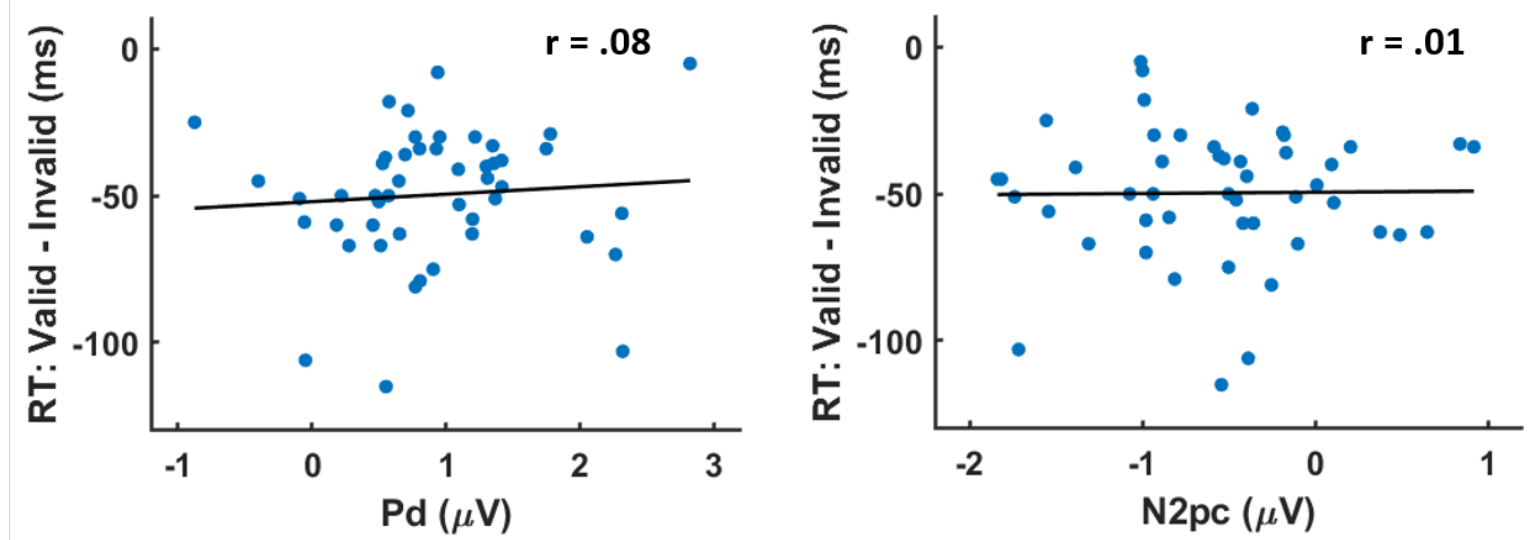
Note. All y-axes show the magnitude of the cueing effect. Attentional capture by target-coloured cues is shown by the blue dots; the SLC produced by nontarget-coloured cues is shown by the red dots. 


\section{Discussion}

Here we sought to examine the source of the same location cost (SLC) by testing the relationship between the magnitude of the SLC as indexed by participants' reaction times, and the amplitude of the $\mathrm{P}_{\mathrm{D}}$ component measured using EEG. The $\mathrm{P}_{\mathrm{D}}$ is commonly taken as an indicator of attentional suppression (Gaspelin \& Luck, 2018b; Sawaki \& Luck, 2010). We reasoned, therefore, that if the SLC is due to attentional suppression of non-target features there should be a positive correlation between the SLC and the magnitude of the $P_{D}$. Alternatively, if the SLC is caused by object-file updating (Carmel \& Lamy, 2014), there should be a negative correlation between the SLC and $P_{D}$, as attentional suppression should lead to weaker stimulus representations which should in turn be easier to update. We reanalysed data from a prior study (Harris et al., 2017) in which we had observed an SLC at the group level but had not analysed the $P_{D}$ component or correlated this with individual differences in the magnitude of the SLC. We observed a robust $P_{D}$ component following all cues. The individual differences analysis supported the object-file updating hypothesis, with a moderate negative correlation observed between the magnitude of the SLC and the $P_{D}$ component produced by nontarget-coloured cues.

In previous work we showed that, under singleton detection mode, increased capture by irrelevant cues is likely caused by a reduction in the SLC (Harris et al., 2019). Although that study did not employ an individual differences approach, the experimental conditions were also associated with an increase in the magnitude of the $P_{D}$ component to irrelevant cues, consistent with the current finding of a reduced SLC with greater $P_{D}$ magnitude. Rather than producing slowing by attentional suppression, it seems that increased suppression (revealed by a larger $P_{D}$ component) serves to diminish the strength of stimulus representations, making subsequent updating of these representations more efficient. Recently, Schoeberl and colleagues $(2018,2020)$ also found support for the object-updating account of the SLC. They found that conditions which would be expected to increase object updating (coloured non-singleton items, rather than achromatic items) at invalid locations served to reduce the SLC, by producing a similar level of slowing at valid compared with invalid locations. This result is somewhat ambiguous, however, as it could plausibly have arisen from feature-specific attentional suppression at the locations of the non-singleton cues. The current findings thus offer important confirmation that attentional suppression is unlikely to drive the SLC.

An alternative account of the SLC suggests that rather than attentional suppression or object file updating, the SLC is due to capture of attention by non-singleton elements in the cue display (Schönhammer et al., 2020). Specifically, this work sought to explain SLCs in prior work on relational capture, in which attention is guided to items that match a specific target-nontarget relation (e.g., the target is redder, or larger, etc..., than the other items). The authors showed that what appeared to be an SLC elicited by cue displays with the opposite relation to the target (e.g., yellower items, in search for a redder target) in fact reflected capture by the relationally matching context (i.e., a 'yellower' singleton is necessarily presented within a 'redder' context). However, this explanation only holds under conditions of relational search, which do not apply in the current study. Recently, a similar suggestion has been put forward to explain the $P_{D}$ component as, in truth, reflecting an inverted N2pc elicited by the non-singleton context elements opposite the distractor (Kerzel \& Burra, 2020, but see Drisdelle \& Eimer, 2021). If this account of the $\mathrm{P}_{\mathrm{D}}$ and the SLC were correct, however, a positive correlation between the two would be expected, as stronger capture by context elements should produce both a larger SLC and a larger $\mathrm{P}_{\mathrm{D}}$ (or inverted N2pc). Our results suggest that one or both of these accounts are incorrect, at least for spatial cueing tasks of the kind used in this and other recent studies (e.g., Carmel \& Lamy, 2014, 2015) .

\section{Conclusion}


The SLC is a well-established phenomenon but somewhat counterintuitive finding in spatial cueing studies. Various accounts of the SLC have been put forward and each has garnered evidence under different stimulus conditions (e.g., Carmel \& Lamy, 2014; Schoeberl et al., 2018; Schönhammer et al., 2020; etc.). Under typical conditions of feature search for colour, increasing evidence supports a postattentional locus of the SLC, potentially due to delays from the updating of object-file properties at the target location (Carmel \& Lamy, 2014). Our results support this conclusion by demonstrating that participants with stronger attentional suppression of irrelevant features (indicated by the magnitude of the $P_{D}$ component) also show a smaller SLC. This result is inconsistent with a suppression account of the SLC, and instead suggests a post-attentional locus of the effect. 


\section{References}

Becker, S. I. (2010). The role of target-distractor relationships in guiding attention and the eyes in visual search. Journal of Experimental Psychology: General, 139(2), 247.

Becker, S. I., Atalla, M., \& Folk, C. L. (2020). Conjunction search: Can we simultaneously bias attention to features and relations?. Attention, Perception, \& Psychophysics, 82(1), 246-268.

Becker, S. I., Folk, C. L., \& Remington, R. W. (2010). The role of relational information in contingent capture. Journal of Experimental Psychology: Human Perception and Performance, 36(6), 1460.

Becker, S. I., Folk, C. L., \& Remington, R. W. (2013). Attentional capture does not depend on feature similarity, but on target-nontarget relations. Psychological Science, 24(5), 634-647.

Becker, S. I., Harris, A. M., Venini, D., \& Retell, J. D. (2014). Visual search for color and shape: When is the gaze guided by feature relationships, when by feature values?. Journal of Experimental Psychology: Human Perception and Performance, 40(1), 264.

Becker, S. I., Harris, A. M., York, A., \& Choi, J. (2017). Conjunction search is relational: Behavioral and electrophysiological evidence. Journal of Experimental Psychology: Human Perception and Performance, 43(10), 1828.

Carmel, T., \& Lamy, D. (2014). The same-location cost is unrelated to attentional settings: An objectupdating account. Journal of experimental psychology: human perception and performance, 40(4), 1465.

Carmel, T., \& Lamy, D. (2015). Towards a resolution of the attentional-capture debate. Journal of experimental psychology: Human Perception and Performance, 41(6), 1772.

Chang, S., \& Egeth, H. E. (2019). Enhancement and suppression flexibly guide attention. Psychological Science, 30(12), 1724-1732.

Chatrian, G. E., Lettich, E., \& Nelson, P. L. (1985). Ten percent electrode system for topographic studies of spontaneous and evoked EEG activities. American Journal of EEG technology, 25(2), 83-92.

Chaumon, M., Bishop, D. V., \& Busch, N. A. (2015). A practical guide to the selection of independent components of the electroencephalogram for artifact correction. Journal of neuroscience methods, 250, 47-63.

Cousineau, D. (2005). Confidence intervals in within-subject designs: A simpler solution to Loftus and Masson's method. Tutorial in Quantitative Methods for Psychology, 1(1), 42-45.

Delorme, A., \& Makeig, S. (2004). EEGLAB: an open source toolbox for analysis of single-trial EEG dynamics including independent component analysis. Journal of neuroscience methods, 134(1), 9-21.

Drisdelle, B. L., \& Eimer, M. (2021). PD components and distractor inhibition in visual search: New evidence for the signal suppression hypothesis. Psychophysiology, e13878.

Eimer, M., Kiss, M., Press, C., \& Sauter, D. (2009). The roles of feature-specific task set and bottomup salience in attentional capture: an ERP study. Journal of Experimental Psychology: Human Perception and Performance, 35(5), 1316.

Folk, C. L., \& Remington, R. (1998). Selectivity in distraction by irrelevant featural singletons: evidence for two forms of attentional capture. Journal of Experimental Psychology: Human perception and performance, 24(3), 847.

Folk, C. L., Remington, R. W., \& Johnston, J. C. (1992). Involuntary covert orienting is contingent on attentional control settings. Journal of Experimental Psychology: Human perception and performance, 18(4), 1030. 
Gaspelin, N., Leonard, C. J., \& Luck, S. J. (2015). Direct evidence for active suppression of salientbut-irrelevant sensory inputs. Psychological science, 26(11), 1740-1750.

Gaspelin, N., Leonard, C. J., \& Luck, S. J. (2017). Suppression of overt attentional capture by salientbut-irrelevant color singletons. Attention, Perception, \& Psychophysics, 79(1), 45-62.

Gaspelin, N., \& Luck, S. J. (2018a). Combined electrophysiological and behavioral evidence for the suppression of salient distractors. Journal of cognitive neuroscience, 30(9), 1265-1280.

Gaspelin, N., \& Luck, S. J. (2018b). The role of inhibition in avoiding distraction by salient stimuli. Trends in cognitive sciences, 22(1), 79-92.

Harris, A. M., Becker, S. I., \& Remington, R. W. (2015). Capture by colour: Evidence for dimensionspecific singleton capture. Attention, Perception, \& Psychophysics, 77(7), 2305-2321.

Harris, A. M., Dux, P. E., Jones, C. N., \& Mattingley, J. B. (2017). Distinct roles of theta and alpha oscillations in the involuntary capture of goal-directed attention. Neuroimage, 152, 171-183.

Harris, A. M., Jacoby, O., Remington, R. W., Travis, S. L., \& Mattingley, J. B. (2019). Taking a closer look at visual search: Just how feature-agnostic is singleton detection mode? Attention, Perception, \& Psychophysics, 81(3), 654-665.

Harris, A. M., Remington, R. W., \& Becker, S. I. (2013). Feature specificity in attentional capture by size and color. Journal of Vision, 13(3), 12-12.

Hickey, C., Di Lollo, V., \& McDonald, J. J. (2009). Electrophysiological indices of target and distractor processing in visual search. Journal of cognitive neuroscience, 21(4), 760-775.

JASP Team (2020). JASP (Version 0.14.1)[Computer software].

Kahneman, D., Treisman, A., \& Gibbs, B. J. (1992). The reviewing of object files: Object-specific integration of information. Cognitive psychology, 24(2), 175-219.

Kerzel, D., \& Burra, N. (2020). Capture by context elements, not attentional suppression of distractors, explains the PD with small search displays. Journal of Cognitive Neuroscience, 32(6), 1170-1183.

Lamy, D., Alon, L., Carmel, T., \& Shalev, N. (2015). The role of conscious perception in attentional capture and object-file updating. Psychological science, 26(1), 48-57.

Lamy, D., Leber, A., \& Egeth, H. E. (2004). Effects of task relevance and stimulus-driven salience in feature-search mode. Journal of Experimental Psychology: Human Perception and Performance, 30(6), 1019.

Luck, S.J., Hillyard, S.A. (1994). Electrophysiological correlates of feature analysis during visual search. Psychophysiology 31, 291-308.

Mertes, C., Wascher, E., \& Schneider, D. (2016). From capture to inhibition: How does irrelevant information influence visual search? Evidence from a spatial cuing paradigm. Frontiers in Human Neuroscience, 10, 232.

Morey, R. D. (2008). Confidence intervals from normalized data: A correction to Cousineau (2005). Tutorial in Quantitative Methods for Psychology, 4(2), 61-64.

Sawaki, R., \& Luck, S. J. (2010). Capture versus suppression of attention by salient singletons: Electrophysiological evidence for an automatic attend-to-me signal. Attention, Perception, \& Psychophysics, 72(6), 1455-1470.

Sawaki, R., \& Luck, S. J. (2013). Active suppression after involuntary capture of attention. Psychonomic bulletin \& review, 20(2), 296-301. 
Schoeberl, T., Ditye, T., \& Ansorge, U. (2018). Same-location costs in peripheral cueing: The role of cue awareness and feature changes. Journal of Experimental Psychology: Human Perception and Performance, 44(3), 433.

Schoeberl, T., Goller, F., \& Ansorge, U. (2020). The influence of display-to-display feature changes on net cueing effects: Evidence for a contribution of object-file updating. Quarterly Journal of Experimental Psychology, 73(6), 908-919.

Schönhammer, J. G., Becker, S. I., \& Kerzel, D. (2020). Attentional capture by context cues, not inhibition of cue singletons, explains same location costs. Journal of Experimental Psychology: Human Perception and Performance, 46(6), 610.

Travis, S. L., Dux, P. E., \& Mattingley, J. B. (2019). Neural correlates of goal-directed enhancement and suppression of visual stimuli in the absence of conscious perception. Attention, Perception, \& Psychophysics, 81(5), 1346-1364.

Vatterott, D. B., \& Vecera, S. P. (2012). Experience-dependent attentional tuning of distractor rejection. Psychonomic bulletin \& review, 19(5), 871-878. 